\title{
Clonidine effects on pain evoked SII activity in humans
}

\author{
Michael Hauck ${ }^{\mathrm{a}}$, Petra Bischoff ${ }^{\mathrm{b}}$, Gunther Schmidt ${ }^{\mathrm{b}}$, Roger Zimmermann ${ }^{\mathrm{a}}$, \\ Juergen Lorenz ${ }^{\mathrm{a}, \mathrm{d}}$, Thomas J. Morrow ${ }^{\mathrm{c}}$, Burkhart Bromm ${ }^{\mathrm{a}, *}$ \\ a Institute of Physiology and Pathophysiology, University of Hamburg, Martinistrasse 52, D-20246 Hamburg, Germany \\ ${ }^{\mathrm{b}}$ Department of Anaesthesiology, University of Hamburg, Martinistrasse 52, D-20246 Hamburg, Germany \\ ${ }^{c}$ Department of Neurology, University of Michigan, Medical Center Drive, Ann Arbor, MI-48109, USA \\ ${ }^{\mathrm{d}}$ Hamburg University of Applied Sciences, Faculty of Life Sciences, Lohbrügger Kirchstraße 65, D-21033 Hamburg, Germany
}

Received 24 February 2005; received in revised form 9 December 2005; accepted 13 December 2005

Available online 24 January 2006

\begin{abstract}
We investigated pain evoked activity in the human secondary sensory cortex (SII) following clonidine administration in six healthy volunteers using multi-channel magnetoencephalography (MEG). Pain was elicited by electrical shocks applied intracutaneously to the fingertip. Subjects rated pain intensity and perceptions of tiredness and passiveness by numerical ranking scales. Each subject underwent two investigations, one week apart from each other, with clonidine doses of $1.5 \mathrm{or} 3.0 \mu \mathrm{g} / \mathrm{kg}$, administered intravenously in a random order and double-blinded. We applied a total number of seven blocks, each consisting of 60 painful stimuli, with one adaptation block, one pre-medication block, four post-medication blocks and one recovery block at the end of the session. MEG data were analysed by dipole reconstruction using CURRY ${ }^{\mathrm{R}}$ (Neuroscan, Hamburg) software package. Cortical activity in the contralateral SII cortex appeared with peak latencies of $118.5 \pm 10 \mathrm{~ms}$. This activity was significantly reduced by clonidine, in parallel with a reduction of pain intensity and enhancement of subjective tiredness and passiveness. There was, however, no significant correlation between MEG and subjective effects. Although both clonidine doses had similar effects, the higher dose induced longer changes. Results indicate that intravenous clonidine is able to relieve pain, but the exact mechanism of clonidine at the level of the SII cortex remains unclear. It is possible that clonidine interacts with the brainstem ascending system regulating vigilance and arousal which would explain the observed decrement of pain induced activity in SII. An additional more specific analgesic action at spinal level cannot be excluded.
\end{abstract}

(c) 2005 European Federation of Chapters of the International Association for the Study of Pain. Published by Elsevier Ltd. All rights reserved.

Keywords: Pain; SII cortex; Clonidine; Vigilance; Analgesia; Dipole reconstruction; MEG

\section{Introduction}

The perception of pain contains sensory, cognitive and affective components (Melzack and Casey, 1967). It is possible to assign some of these components to circumscript activities in different brain regions (Talbot et al., 1991; Casey et al., 1996; Bromm et al., 2000).

\footnotetext{
${ }^{*}$ Corresponding author. Tel.: +49 4042803 6170; fax: +494042803 7752.

E-mail address: bromm@uke.uni-hamburg.de (B. Bromm).
}

According to their main neuronal input from lateral thalamic nuclei, the primary (SI) and secondary somatosensory cortices (SII) are parts of the lateral pain system (Treede et al., 2000) which governs mainly sensory-discriminative functions, i.e., the processing of information about duration, strength and location of pain. In contrast, the cingulate gyrus is part of the medial pain system and plays an important role in the cognitive and affective aspects of pain perception (Bush et al., 2000). Most knowledge about SII comes from invasive animal studies focussing on tactile functions (for review see

1090-3801/\$32 ㄷ 2005 European Federation of Chapters of the International Association for the Study of Pain. Published by Elsevier Ltd. All rights reserved.

doi:10.1016/j.ejpain.2005.12.003 
Burton et al., 1982). With the introduction of biomagnetometry, the human SII cortex can be investigated noninvasively for the first time, especially in the context of pain (Hari et al., 1983a,b; Laudahn et al., 1995; Kakigi et al., 2000). Pain relevant SII areas are located superficially in the cortex, and the respective columns show a tangential direction to the head surface, allowing the detection of relatively large magnetic fields during neuronal activity (see, for example, Hillebrand and Barnes, 2002). Pain related SII activity has meanwhile been described by many authors using functional brain imaging methods, in particular multi channel MEG recordings. The activity starts between 90 and $150 \mathrm{~ms}$ after the pain stimulus, depending on the body site and activated nerve fibre spectrum (Howland et al., 1995; Bromm et al., 1996; Kakigi et al., 1996; Lenz et al., 1998; Frot et al., 1999; for review see Bromm and Lorenz, 1998). Also, it is well known that pain-induced SII activity depends strongly on the vigilance level and the attentional state of the brain (Bromm et al., 2000; Hämäläinen et al., 2000; Nakamura et al., 2002).

The following study addresses the question whether pain evoked SII activity is altered by clonidine, an $\alpha 2$-agonist with both antinociceptive and sedative properties. Clonidine is typically used as co-analgesic in postoperative pain management (see, e.g., Bischoff and Kochs, 1993; Kanui et al., 1993; Eisenach et al., 2000). Using dipole reconstruction analysis of magnetic brain maps gathered from multichannel MEG, we investigated the effects of two different doses of intravenously administered clonidine on the SII-Cortex.

\section{Methods}

\subsection{Subjects and test procedure}

The study was performed on seven healthy male students (six right handed, one left handed) with written informed consent (age $26 \pm 3$ years, height $183 \pm 7 \mathrm{~cm}$, weight $76 \pm 5 \mathrm{~kg}$ ) after being approved by the Institutional Ethics Committee. Control of standard clinical and neurological status, serum chemistry, and MRI scan of the brain yielded normal findings and there was no history of neurologic or psychiatric abnormalities. The MRI scan was additionally used to calculate the dipoles in the individual anatomy. One subject was discarded, because of strong artefacts during MEG recordings. We tested subjects in two separate sessions, one week apart from each other, with two doses of clonidine $(1.5$ and $3.0 \mu \mathrm{g} / \mathrm{kg})$, administered intravenously. The order of doses was randomized and double-blinded. Subjects underwent a clinical examination before and $3 \mathrm{~h}$ after medication in which blood pressure, blood oxygenation and heart rate was monitored (Jenacor, Jena, Germany). Pain was induced according to the intracuta- neous pain model (Bromm and Meier, 1986; Scharein and Bromm, 1998). Briefly, a thin needle electrode was fed through a hole punched into the horny skin of the tip of the middle finger of the non-dominant hand and an electrical pulse ( $5 \mathrm{~ms}$ duration) delivered to the nearest neighbourhood of nociceptors. We applied two different stimulus intensities according to $2\left(I_{1}\right)$ and $3\left(I_{2}\right)$ times individual pain threshold $\left(I_{\mathrm{p}}\right)$ the latter being determined by a series of increasing stimulus intensities and decreasing stimulus intensities at the beginning of each session. The subjects had to rate the stimulus intensity according to a numerical ranking scale (NRS) with values from 0 to 10 . Values of more than three described a painful sensation and determined the pain threshold $I_{\mathrm{p}}$. The average pain threshold intensity was $0.2 \mathrm{~mA}$ ( $\pm 0.07 \mathrm{~mA}$ standard deviation).

During the experiments subjects lay in a noisereduced, electrically and magnetically shielded chamber containing the cryostat supplied with 31 gradiometers. The stimuli were given in blocks of 60 with the two intensities $I_{1}$ and $I_{2}$ in a quasi random sequence; the inter-stimulus interval varied between 6 and $10 \mathrm{~s}$. Thus each block lasted approximately $12 \mathrm{~min}$. Recordings of the single trials were averaged within each block as average block data to reduce the signal to noise ratio. On each investigation day, seven blocks of stimuli were applied with intervals of 3-5 min between the first six blocks. The first one (block 0) was done for adaptation only and excluded from further evaluation, to minimize the largest source of variability due to habituation (Bromm and Lorenz, 1998). The next block served to achieve pre-medication values (block PRE). Immediately after administration of clonidine, six consecutive blocks explored the effects over a post-medication time period lasting from $0 \mathrm{~min}$ (POST1) to $55 \mathrm{~min}$ (POST4) after medication. At the end of the session ( 90 min after medication) the last stimulus block was performed (RECOVERY). Three seconds after each pain stimulus, a tone prompted the subjects to rate their sensation using the NRS. Moreover, subjective tiredness and passiveness was measured by visual analog scales $(0-100)$. Higher values describe a greater extent of tiredness and passiveness, whereas values around 0 describe no tiredness and passiveness at all. The subjects were asked to describe their perceptions before the first block as well as 60 and $120 \mathrm{~min}$ after clonidine injection.

\subsection{Data recording and evaluation}

MEG data were measured with a 31-channel biomagnetometer (Philips, Hamburg); the gradiometers were centred over the hemisphere contralateral to the stimulated side (C3, C4). A 32 channel EEG was also recorded to control for changes in vigilance. Responses were restricted to a frequency band between 0.3 and $100 \mathrm{~Hz}$ and digitized at a sampling rate of $200 \mathrm{~Hz}$. A 
spherical model obtained from the individual MRI scans was fitted to the head shape of each subject. Further dipole reconstructions were calculated with the CUR$\mathrm{RY}^{\mathrm{R}}$ software package (Neuroscan, Hamburg). After visual artifact control (on the average less than $10 \%$ of all recordings were discarded), $70 \mathrm{~Hz}$ low pass filtering and common mode rejection, data of the pre-medication blocks were subjected to the one moving dipole approximation, which is sufficient for SII evaluations as determined by earlier studies (Bromm and Lorenz, 1998). With a relative maximum of the mean global field power (GFP) between 80 and $160 \mathrm{~ms}$, the best fitting dipole had to fulfil the following attributes: a goodness of fit (GOF) better than $90 \%$, a spatial stability $(D)$ lower then $10 \mathrm{~mm}$, and a lifetime of at least $15 \mathrm{~ms}$. Spatial stability $(D)$ describes the euclidean distance of the dipole coordinates between adjacent sample points. Life-time means that these parameters have to occur for at least $15 \mathrm{~ms}$. If these attributes were fulfilled, the dipole could be regarded as stable over time. As a last step, all dipole coordinates were transformed into the Talairach coordinate system (Talairach and Tournoux, 1988), using the individual head morphology.

The dipole found in the MEG data block PRE was then fixed for analysis of the cortical activity after clonidine administration. This had to be done, since the evoked fields were drastically reduced under clonidine with a low signal to noise ratio. Moreover, we did not assume that clonidine shifts the location of pain-induced SII activity. For further statistical analysis SPSS 10.0 was used (SPSS, Chicago, IL). After first testing all data for normal distribution using a Kolmogorov-Smirnov test, the global data set was subjected to a two factorial analysis of variance (ANOVA). Significant results were further analyzed with paired student $t$-tests for dependent samples. Correlations between SII dipole strength and pain perception were calculated as well.

\section{Results}

Results of a single subject (HE) with the original recorded data of the pre-medication block are shown as butterfly plot in Fig. 1A. It illustrates a maximum of evoked magnetic activity at approximately one hundred ms after stimulus onset. Fig. 1B displays the isocontour map of this field at peak maximum of $105 \mathrm{~ms}$ projected on the individual's head (obtained from the MRI scans). It indicates a distinct inversion in polarity (continuous lines indicate outward fields, dashed lines field entrances) that is characteristic for a dipolar field. Source reconstruction of this evoked field revealed a dipole, the location of which is shown in Fig. 1C. This dipole had a maximal dipole strength $(Q=29.1 \mu \mathrm{A} \mathrm{mm})$ at $105 \mathrm{~ms}$ and a goodness of fit (GOF) of $98.3 \%$ between measured and calculated fields around peak latency

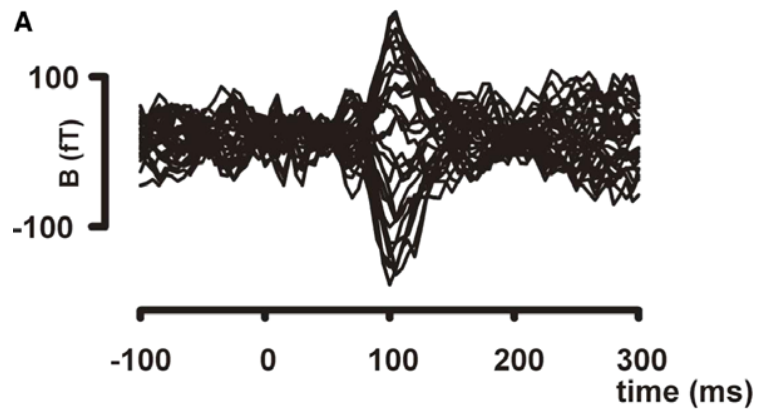

B

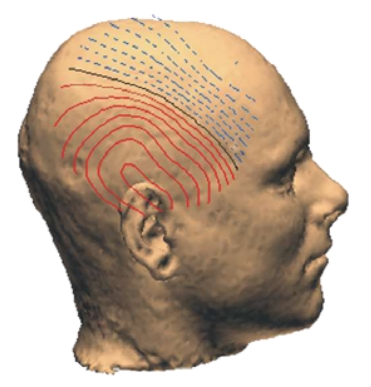

map $105 \mathrm{~ms}$

C

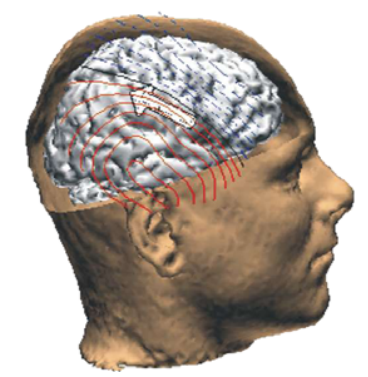

dipole 105

Fig. 1. Data of the premedication block, single subject HE. Above (A) is shown the butterflyplot of all 31 MEG channels with two field maxima. The isocontour map of the second maxima after $105 \mathrm{~ms}$ is shown in the middle (B) and projected on the subjects head. Dipole reconstruction of this field with interindividual anatomy parameters found a dipole in SII (C).

time. The coordinates of the identified dipole in the Talairach system were: $X=54.1 ; Y=-9.0 ; Z=22.6$, which is clearly in the SII area of the dominant hemisphere (Table 1) The dipole direction was parallel to the cortex surface and oriented from superior to ventral. It should be noted that a similar dipole appears in the ipsilateral hemisphere (bilateral SII co-activation; Kakigi et al., 1995; Bromm et al., 2000) but cannot be identified with only one cryostat positioned over the $\mathrm{C} 3$ location of the 10-20 system, as used in this study.

Similar findings were made in all subjects regarding the pre-medication blocks of the study. The peak maxima in mean global fields varied from subject to subject in a range between 90 and $130 \mathrm{~ms}$. Results are given in Fig. 2, illustrating the high re-detection accuracy of measurements. In principle, all dipoles were found in SII; only one of the 14 pre-medication blocks (subject PR, day 2) failed to be localized within SII. The dipole coordinates and additional parameters are summarised 
Table 1

Dipole coordinates in the Talairach-system $(x, y, z)$; dipole strength $(Q)$; mean global field maxima (MGF); goodness of fit (GOF) and the latency time (time) are shown for both medication conditions $(\mu \mathrm{g} / \mathrm{kg})$ for each subject at the premedication block

\begin{tabular}{|c|c|c|c|c|c|c|c|c|}
\hline Subject & $(\mu \mathrm{g} / \mathrm{kg})$ & $x(\mathrm{~mm})$ & $y(\mathrm{~mm})$ & $z(\mathrm{~mm})$ & $Q(\mu \mathrm{A} \mathrm{mm})$ & GOF $(\%)$ & MGF (fT) & Time (ms) \\
\hline $\mathrm{HE}$ & 3 & 54.1 & -9 & 22.6 & 29.1 & 98.3 & 134 & 100 \\
\hline $\mathrm{HE}$ & 1.5 & 41.7 & -16.3 & 27.7 & 21.8 & 98.7 & 92.5 & 105 \\
\hline $\mathrm{ME}$ & 3 & 61.9 & -16.6 & 9.1 & 11.8 & 96.1 & 61 & 120 \\
\hline ME & 1.5 & 55.5 & -11.6 & 5.2 & 27.3 & 97.3 & 106 & 120 \\
\hline PR & 3 & 55.3 & 10.8 & 32.5 & 8.84 & 93.8 & 64.4 & 130 \\
\hline PR & 1.5 & & & & & & & \\
\hline SA & 3 & 35.5 & -5.6 & 16.3 & 44.4 & 98.7 & 118 & 105 \\
\hline SA & 1.5 & 37.4 & -4.8 & 21.2 & 29.1 & 98.6 & 84 & 115 \\
\hline WA & 3 & 58.8 & -5.1 & 38.3 & 8.05 & 86.9 & 46.1 & 125 \\
\hline WA & 1.5 & 56 & -1.5 & 26.8 & 6.2 & 83.1 & 31.8 & 125 \\
\hline KU & 3 & $(-) 60.1$ & -3.9 & 17.3 & 35.2 & 97.6 & 139 & 125 \\
\hline KU & 1.5 & $(-) 54.4$ & -10.4 & 13.1 & 33.2 & 98.8 & 128 & 125 \\
\hline Mean & & 52.2 & -5.3 & 21.9 & 22.57 & 91.75 & 84.61 & 118.75 \\
\hline SD & & 8.8 & 8.9 & 10.1 & 12.38 & 13.17 & 42.26 & 10.25 \\
\hline
\end{tabular}

Values are the mean value with standard deviation.

in Table 1. In general, the intra-individual dipole site variations were within the spatial resolution of our techniques $(<6 \mathrm{~mm}$, for further details see Fuchs et al., 1998).

The effects of clonidine are shown in Fig. 3 for subject HE. Already $20 \mathrm{~min}$ after medication, within stimulus block POST2, pain induced SII activity disappeared completely. As a consequence the signal to noise ratio was very low after clonidine application in all subjects. In order to calculate a reasonable dipole model, we had to apply the fixed dipole approximation (see methods) with the individual coordinates given in Table 1 ; in case of PR, day 2, we used those coordinates found in day 1. In this way the clonidine induced reduction in SII dipole-strength was quantified. The effects of the two clonidine doses upon the SII dipole strengths are shown as a function of medication time in Fig. 4A. At the higher dose of clonidine. the dipole strength decreased from $22.9 \pm 15.4 \mu \mathrm{A} \mathrm{mm}$ in the PRE block to $19.2 \pm$ $14.5 \mu \mathrm{A} \mathrm{mm}$ in POST block 1 and $5.5 \pm 14.5 \mu \mathrm{A} \mathrm{mm}$ in POST block2, which was $22 \%$ of the PRE value. Post Block 3 and 4 and the REC Block yielded a gradual recovery of the dipole strength to mean values of $7.9 \pm 4.3$, $13.1 \pm 11.4$ and $11.4 \pm 9.0 \mu \mathrm{A} \mathrm{mm}$, respectively. A

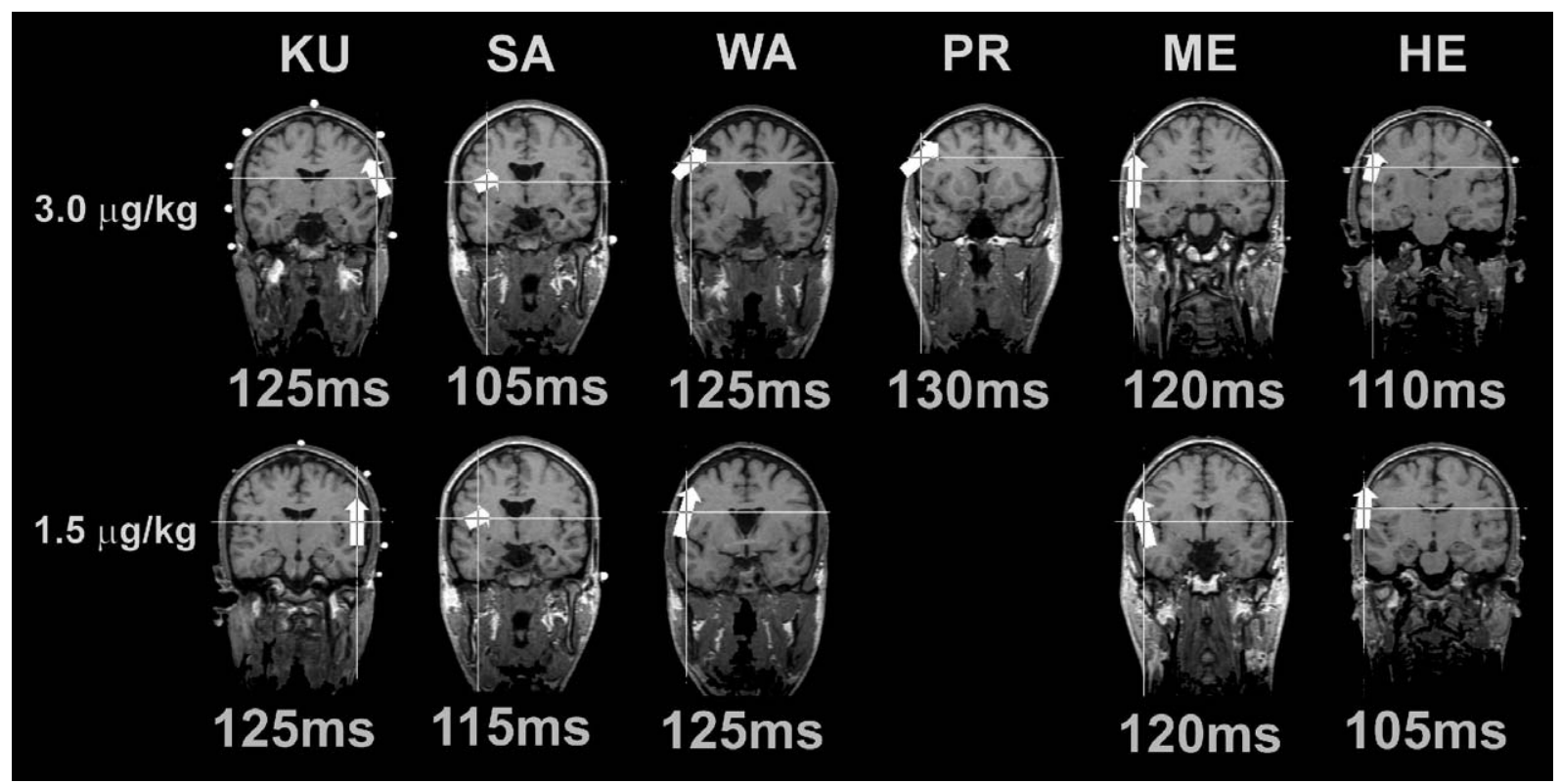

Fig. 2. Dipole localisations of all subjects were found in SII. It is only shown the premedication block of the experiment conditions with higher $(3.0 \mu \mathrm{g} / \mathrm{kg})$ and lower $(1.5 \mu \mathrm{g} / \mathrm{kg})$ clonidine dose, which was applicated after this block. These interindividual dipole localisations were fixed for further source analysis to investigate the effect of clonidine. Subject KU was left handed, so the dipole was found in the contralateral side. 


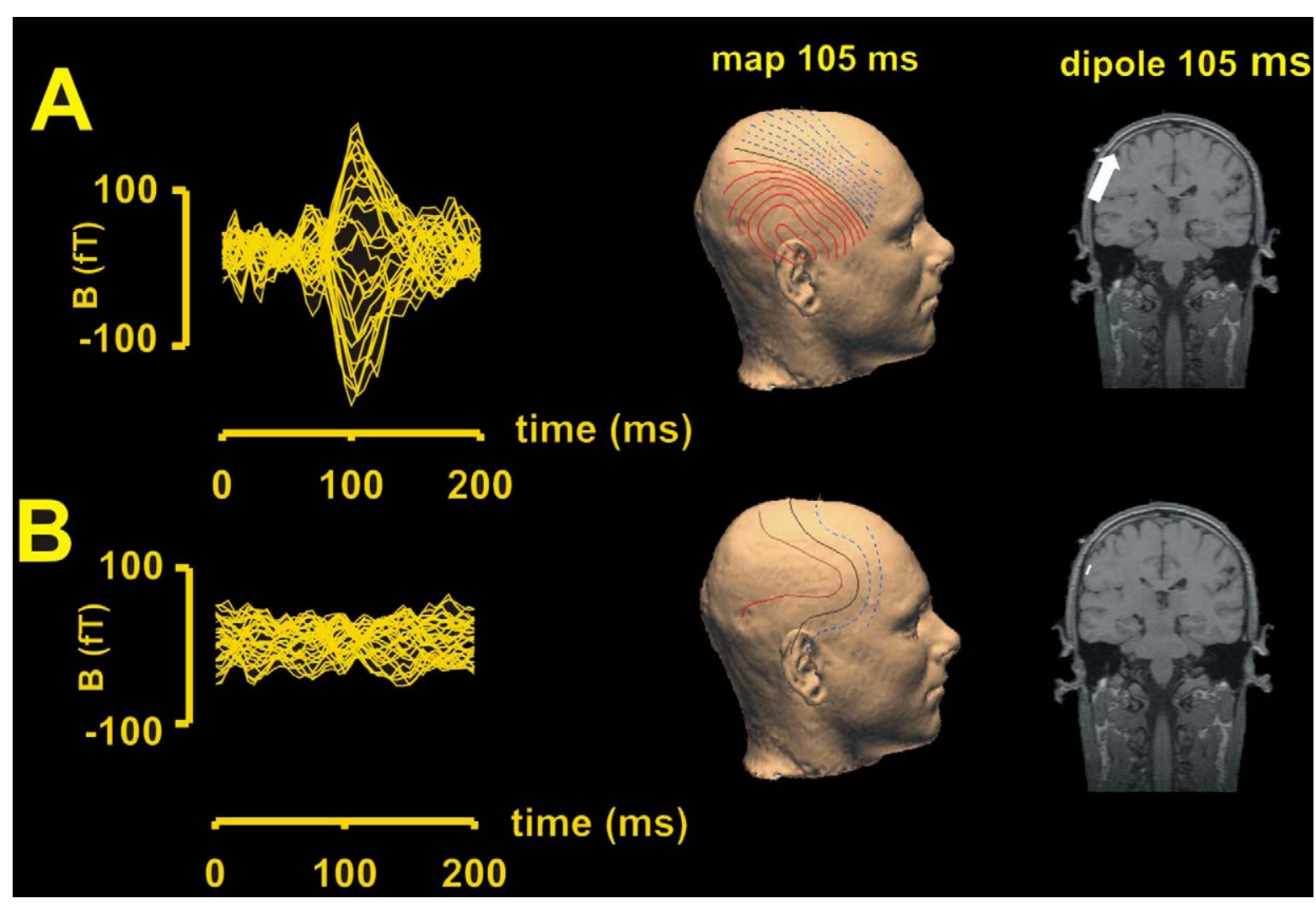

Fig. 3. Butterfly plots with corresponding iso contourmaps and dipole localisations are shown for the premedication block (A) and 20 min after clonidine application (B). The SII-activity is reduced after clonidine application. Shown is subject HE.

similar time course of change occurred for the lower dose. The dipole strength decreased from $22.2 \pm 9.9 \mu \mathrm{A} \mathrm{mm}$ in the PRE block to $13.6 \pm 10.0 \mu \mathrm{A} \mathrm{mm}$ in POST block1, to $6.8 \pm 5.1 \mu \mathrm{A} \mathrm{mm}$ in POST block2 and to $6.4 \pm 2.9 \mu \mathrm{A} \mathrm{mm}$ in POST block3, the latter being $28 \%$ of the PRE value. Again, there was a recovery of the dipole strength to $7.7 \pm 5.6 \mu \mathrm{A} \mathrm{mm}$ in POST block4 and $16.9 \pm 14.6 \mu \mathrm{A} \mathrm{mm}$ in the REC block. Thus, clonidine dose had no effect on the reduction of the SII dipole strength, but appeared to extend its duration.

Fig. 4B shows the pain ratings for all subjects, which were measured during the treatments in each individual, as described above. At the higher dose of clonidine, pain ratings decreased from $4.7 \pm 0.7$ in the PRE block to $3.6 \pm 0.4$ in POST block1 and $2.3 \pm 1.6$ in POST block2, which was $48 \%$ of the PRE value followed by a recovery of the pain ratings to $2.6 \pm 1.1$ in POST block3, $3.4 \pm 0.8$ in POST block 4 and $4.2 \pm 0.4$ in the REC block. For the lower clonidine dose pain ratings decreased from $4.2 \pm 0.8$ in the PRE block to $4.2 \pm 0.9$ in POST block1 and $2.3 \pm 1.4$ in POST block2, which was $53 \%$ of the PRE value. Again, there was a recovery of the pain ratings to $3.2 \pm 1.1$ in POST block3, $3.6 \pm 0.5$ in POST block 4 and $4.7 \pm 1.6$ in the REC block. Although reductions of pain ratings generally corresponded well to the reduction of SII dipole strength, but the time course of pain ratings failed to indicate a prolonged action of the higher clonidine dose. Further statistical analysis substantiated these findings. The results of the ANOVA (Table 2) yielded significant block effects, for both the dipole strength $(F=8.7$; $p<0.05)$ and pain ratings $(F=7.2 ; p<0.05)$, but no main or interaction effects occurred with dose on dipole strength or pain ratings. Subsequent $t$-test analysis of significant ANOVA effects is displayed in Table 3. When compared with the pre-Block significant reductions of SII-dipole strength (Table 3A) and pain ratings (Table 3B) occurred for both clonidine doses in POST blocks 2, 3 and 4. The failure of change in post Block 1 is in agreement with the pharmacokinetics of clonidine (Bischoff et al., 2000). The half maximum value of clonidine serum concentration is not reached before $10 \mathrm{~min}$. No correlations were found between dipole strengths $(Q)$ and pain ratings (Table $3 \mathrm{C}$ ) with the one exception, subject KU.

Further analysis of the subjective effects of clonidine revealed an enhancement of tiredness and passiveness during maximal clonidine concentration (Fig. 5). During baseline the subjects rated their tiredness as $24.5 \pm 18.1$. Sixty minutes after medication the tiredness was enhanced to $63.3 \pm 34.3$ ( $258 \%$ of start value) which lasted until $120 \mathrm{~min}$ after clonidine administration with 

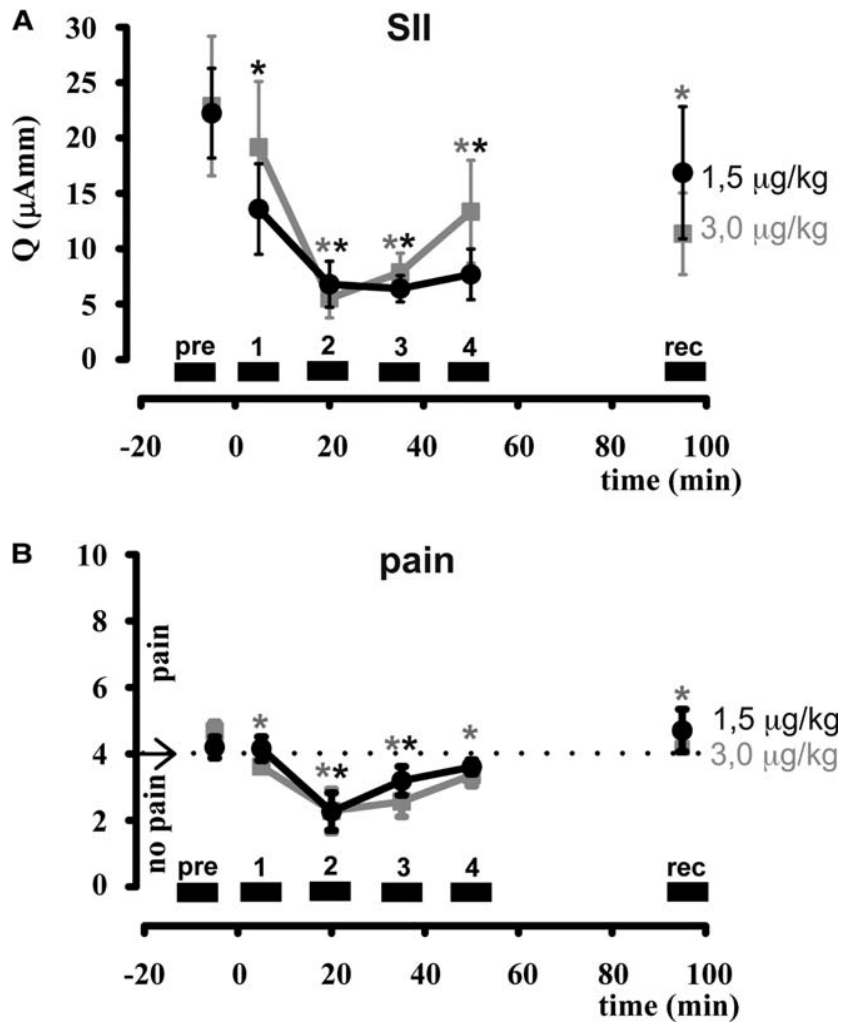

Fig. 4. Mean dipole strength values (A) and the pain ratings (B) with standard error of the mean over all subjects. The dipole was fixed in the individual SII area for each subject (see Fig. 2). It is shown, that SIIactivity and the pain ratings are attenuated after clonidine application.

Table 2

Results of the ANOVA for the dipole strength (A) and pain ratings (B)

\begin{tabular}{lccrr}
\hline & $\mathrm{df}$ & $F$ & \multicolumn{1}{c}{$p$} & $p[\varepsilon]$ \\
\hline A. Dipole strength & & & & \\
Block & 5 & 8.868 & $<0.001$ & 0.008 \\
Concentration & 1 & 0.033 & 0.297 & 0.609 \\
B. Pain rating & & & & \\
Block & 5 & 7.244 & $<0.001$ & 0.026 \\
Concentration & 1 & 0.318 & 0.597 & 0.597 \\
\hline
\end{tabular}

A significant block effect $(p<0.001)$ with Greenhouse-Geisser-correction $(p[\varepsilon]<0.008)$ for the dipole strength and the pain ratings $(p<0.001 ; p[\varepsilon]<0.026)$ was found. No significant effect for concentration was found either for the dipole strength $(p=0.609)$ nor for the pain ratings $(p=0.597)$.

the value of $51.5 \pm 22.8$. The passiveness was rated as $38.7 \pm 23.8$ before clonidine administration and changed to $59.3 \pm 28.3$ after $60 \mathrm{~min}(152.3 \%$ of the PRE value) and $51.5 \pm 30.7$ after $120 \mathrm{~min}$. Before low dose clonidine administration the subjects rated their tiredness as $37.1 \pm 32.5$. After $60 \mathrm{~min}$ the tiredness was enhanced to $64.5 \pm 24.9$ ( $171 \%$ of PRE value) which recovered after $120 \mathrm{~min}$ to values of $32.2 \pm 27.7$. The passiveness was rated as $33.0 \pm 22.0$ before clonidine administration and changed to $50.8 \pm 32.2$ after $60 \min (153.4 \%$ of the PRE value) and $32.5 \pm 23.5$ after $120 \mathrm{~min}$.
Table 3

Results of the $t$-tests for the dipole strength (A) and the pain ratings (B)

\begin{tabular}{|c|c|c|c|c|}
\hline & \multicolumn{2}{|c|}{$1.5 \mu \mathrm{g} / \mathrm{kg}$ Clonidine } & \multicolumn{2}{|c|}{$3.0 \mu \mathrm{g} / \mathrm{kg}$ Clonidine } \\
\hline & $t$-Value & $p$-Value & $t$-Value & $p$-Value \\
\hline \multicolumn{5}{|c|}{ A. Dipole strength } \\
\hline Pre-post 1 & 2.69 & 0.021 & 1.17 & 0.147 \\
\hline Pre-post 2 & 2.83 & 0.018 & 3.19 & 0.012 \\
\hline Pre-post 3 & 4.72 & 0.002 & 3.03 & 0.015 \\
\hline Pre-post 4 & 3.81 & 0.006 & 2.21 & 0.039 \\
\hline Pre-rec & 1.09 & 0.161 & 2.86 & 0.018 \\
\hline \multicolumn{5}{|l|}{ B. Pain rating } \\
\hline Pre-post 1 & 0.14 & 0.445 & 3.44 & 0.009 \\
\hline Pre-post 2 & 2.75 & 0.02 & 2.70 & 0.022 \\
\hline Pre-post 3 & 1.86 & 0.061 & 3.47 & 0.009 \\
\hline Pre-post 4 & 1.42 & 0.107 & 3.22 & 0.011 \\
\hline \multirow[t]{2}{*}{ Pre-rec } & -1.02 & 0.178 & 1.68 & 0.076 \\
\hline & $r$-Value & $p$-Value & $n$-Value & \\
\hline \multicolumn{5}{|l|}{ C. Correlation } \\
\hline $\mathrm{HE}(Q * \mathrm{PR})$ & 0.453 & 0.139 & 12 & \\
\hline $\mathrm{KU}(Q * \mathrm{PR})$ & 0.942 & $<0.001$ & 12 & \\
\hline $\operatorname{ME}(Q * \mathrm{PR})$ & 0.499 & 0.099 & 12 & \\
\hline $\mathrm{PR}(Q * \mathrm{PR})$ & 0.24 & 0.524 & 12 & \\
\hline $\mathrm{SA}(Q * \mathrm{PR})$ & 0.361 & 0.248 & 12 & \\
\hline WA $(Q * \mathrm{PR})$ & 0.46 & 0.886 & 12 & \\
\hline
\end{tabular}

Clonidine reduces the SII-dipole strength and the pain ratings. A correlation $(C)$ between the dipole strength $(Q)$ and the pain rating (PR) for the single subjects was only found for subject KU.

\section{Discussion}

By using an experimental pain model in six healthy volunteers in combination with MEG measurements this study demonstrates a substantial reduction of pain intensity in parallel with decreased evoked activity in the secondary somatosensory cortex (SII) following administration of clonidine, at doses of 1.5 and $3.0 \mu \mathrm{g} /$ $\mathrm{kg}$ body weight. Dipole reconstruction based on the individual brain morphology localized the induced magnetic activity maximum within a latency range from 90 to $120 \mathrm{~ms}$ reliably within the SII cortex in all subjects. The intraindividual redetection accuracy was below $6 \mathrm{~mm}$, which is the spatial resolution limit of our measurements and calculations. Although both subjective and physiological parameters of pain decreased after clonidine they were not statistically correlated. This supports the idea that stimulus induced SII activity does not necessarily reflect the painfulness of stimulation. Furthermore, we could not find any differences according to the two doses of clonidin, which could indicate a ceiling effect on SII activity. Using the lower dose of $1.5 \mu \mathrm{g} /$ $\mathrm{kg}$, the SII activity was already reduced to a minimum value near the level of background noise as early as after 20 min post medication, from which no further reduction is possible. On the other hand the time course of changes suggests a longer reduction of pain-induced SII activity for the higher compared to the lower 

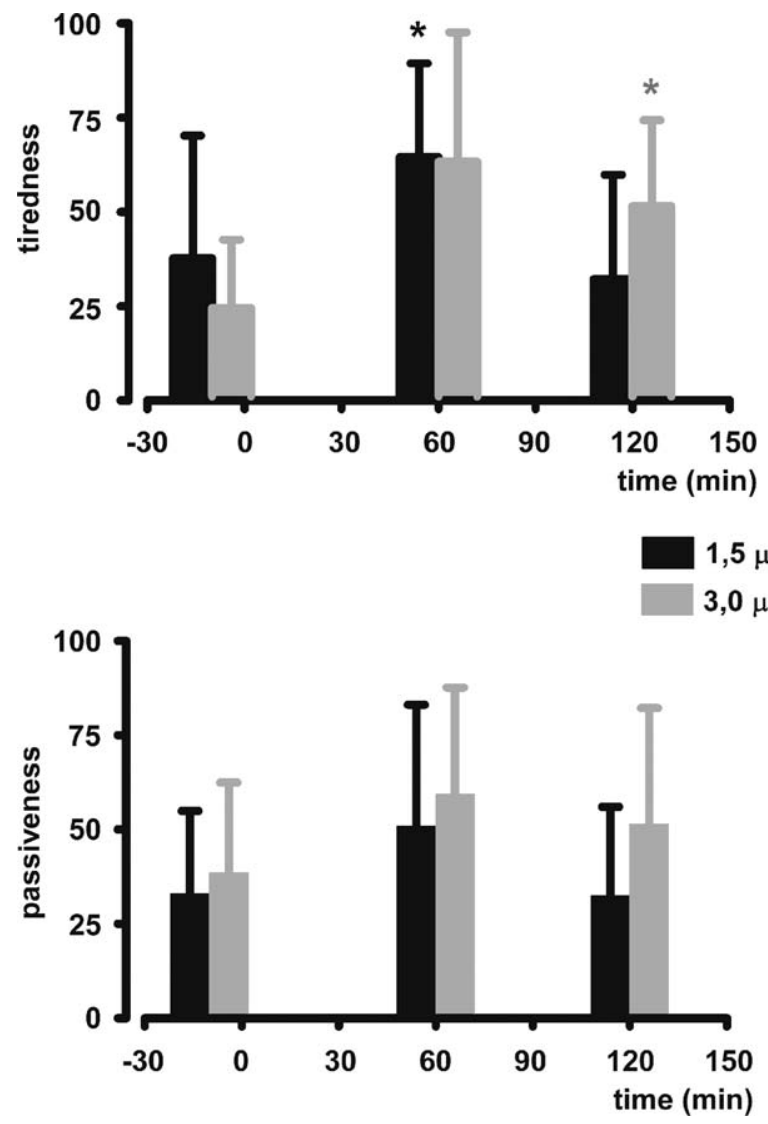

Fig. 5. Results of the questionaire dealing with the individual feeling of tiredness (above) and passiveness (below) of all subjects, $10 \mathrm{~min}$ before the experiment and 60 and 120 min after it.

clonidine dose, compatible with the pharmakokinetics of clonidine and findings of other authors (Bischoff et al., 2000, 2004).

Pain was induced by brief electrical current pulses, which were applied intracutaneously by a tiny hole through the horny layer of the middle fingertip, thus delivering the stimulus in the nearest neighbourhood of cutaneous nociceptors. This model induces a distinct pinprick pain and has shown its usefulness in many experiments measuring pain and pain relief under efficient analgesics (for review see Bromm and Lorenz, 1998). But all experimental pain models co-activate receptors of the tactile system as well, maybe also the infrared laser heat pulse (Treede et al., 1995). There is no doubt that tactile information reaches primary and secondary somatosensory areas. Therefore experimental pain-inducing stimuli always activate the SII cortex to a higher or lower degree, as well as non-nociceptive afferents, thus activation of SII is not necessarily a sign for pain experience (for a detailed discussion see Bromm et al., 2000).

Earlier studies of our group demonstrated the effects of habituation and morphine on subjective pain and pain evoked SII activity using the same intracutaneous pain model in a placebo-controlled design (Quante et al., 2004). Given these results that yielded only small placebo effects the investigated decrease of pain evoked activity during the present study is likely due to the administration of clonidine even though we decided not to involve a placebo control in this study for procedural reasons.

The observed reduction of experimental pain in this study is consistent with clinical reports. Although placebo experiments are necessary to document new properties of drugs, and would have complemented our findings for the purpose of this study, pre-post comparisons seemed sufficient. Furthermore these experiments are extremely expensive and the mean values and normative variances of dipole identifications in SII are well known within and between subjects from a huge number of preceding experiments using the same standardized design (Lorenz et al., 2005; Nakamura et al., 2002; Bromm, 2001). However, the effects due to the long session duration are in the order of $5-8 \%$. Because of this, it is clear that clonidine in both concentrations attenuates the SII activity drastically by $50 \%$ or more. Thus, the question is how clonidine interacts so strongly with pain perception. For example, in postoperative pain management intrathecal application of clonidine is used successfully to reduce the dose of morphine (Eisenach et al., 2000; Kanui et al., 1993; for review see Bischoff and Kochs, 1993). Studies in animals and patients have shown that the transdermal, epidural and intravenous administration of clonidine reduces pain intensity in neuropathic pain syndromes for periods varying from some hours up to 1 month. However, there is little evidence for a specific antinociceptive effect of intravenous administered clonidine (Bernard et al., 1995; Eisenach et al., 1998; Hao et al., 1996). These authors conclude that the clonidine-related decrease in pain experience depends on the level of arousal and is most probably not due to direct actions on the nociceptive system (see also Kakigi et al., 1996; Hämäläinen et al., 2000; Höchstetter et al., 2000; Nakamura et al., 2002). On the other hand, it is well known that SII activity depends on the vigilance level and on attentional states of the subject under investigation (Mima et al., 1998; Sarter et al., 2001; Nakamura et al., 2002). Obviously the SII cortex is tonically pre-primed by subcortical regions, which control the state of vigilance and arousal. Sedatives, tranquilizers and hypnotics attenuate stimulus evoked SII activity as well as mental distraction from the stimulus event. Also clonidine is well known to reduce vigilance and arousal (Bischoff and Kochs, 1993; Coull et al., 1995; Clark et al., 1986). Analysis of the spontaneous electroencephalogram (EEG) supports these observations. Clonidine attenuates the alpha rhythm and increases slow theta and delta rhythms (Bischoff et al., 2000), parameters that are well known to correlate with reaction time and subjective fatigue. A limitation of our study is that we only evaluated sub- 
jective perceptions like tiredness and passiveness without raising reaction times or other clinical parameters of sedation and directly relate this to the action of clonidine on the SII cortex. For this reason we cannot clearly differentiate between specific and unspecific modulations of pain induced SII activity, a question that need to be addressed in further studies with greater focus on multimodal assessment of sedation in comparison with MEG and EEG. Nevertheless, we were able to show, that clonidine decreases pain ratings and pain evoked SII activity allowing the conclusion that intravenous clonidine, and not only intrathecal clonidine, is able to relieve pain, but the detailed cortical mechanisms of intravenous clonidine on the pain system remains unclear.

\section{Conclusions}

Intravenous administration of clonidine was able to reduce pain evoked SII activity (decrease from PRE = $100 \%$ to POST $2=22 \%$ ) and pain ratings (decrease from PRE $=100 \%$ to POST2 $=48 \%$ ), without significant correlation between these parameters. Similarly an increase of tiredness (258\%) and passiveness $(152.3 \%)$ was observed. Therefore, not only the intrathecal, but also the intravenous application of clonidine suppresses pain. Given the close association of pain perception and vigilance, the observed attenuation of pain evoked responses, represented at the level of the SII cortex, can be explained by a reduction of vigilance and arousal, effects of clonidine that have been reported earlier and something for which clonidine is well known. Further studies using physiological indicators of sedation in combination with those of pain can better discriminate the relative contribution of unspecific sedative and specific analgesic clonidine actions.

\section{Acknowledgements}

We gratefully acknowledge the technical support of Gerd Steinmetz, Krimhild Saha and the department of Radiology of the University of Hamburg.

\section{References}

Bernard JM, Kick O, Bonnet F. Comparison of intravenous and epidural clonidine for postoperative patient-controlled analgesia. Anesthes Analg 1995;81(4):706-12.

Bischoff P, Kochs E. Alpha 2-agonists in anesthesia and intensive medicine. Anästh Intensiv Notf Schmerzther 1993;28(1):2-12.

Bischoff P, Scharein E, Schmidt GN, von Knobelsdorff G, Bromm B, Schulte am Esch JS. Topography of clonidine-induced electroencephalographic changes evaluated by principal component analysis. Anesthesiology 2000;92(6):1545-52.
Bischoff P, Schmidt GN, Scharein E, Bromm B, Schulte am Esch J. Clonidine induced sedation and analgesia - an EEG study. J Neurol 2004;251(2):219-21.

Bromm B. Brain images of pain. News Physiol Sci 2001;16:244-9.

Bromm B, Meier W. The intracutaneous stimulus: a new pain model for algesimetric studies. Method Find Exp Clin Pharmacol 1986;6(7):405-10.

Bromm B, Lorenz J. Neurophysiological evaluation of pain. Electroen Clin Neurophysiol 1998;107(4):227-53.

Bromm B, Lorenz J, Scharein E. Dipole source analysis of brain activity in the assessment of pain. Recent Adv Clin Neurophysiol 1996;1:328-55.

Bromm B, Scharein E, Vahle-Hinz C. Cortex areas involved in the processing of normal and altered pain. Prog Brain Res 2000;1:129-32.

Burton H, Mitchell G, Brent D. Second somatic sensory area in the cerebral cortex of cats: somatotopic organization and cytoarchitecture. J Comp Neurol 1982;210(2):109-35.

Bush G, Luu P, Posner MI. Cognitive and emotional influences in anterior cingulate cortex. Trends Cogn Neurosci 2000;4(6):215-22.

Casey KL, Minoshima S, Morrow TJ, Koeppe RA. Comparison of human cerebral activation pattern during cutaneous warmth, heat pain, and deep cold pain. J Neurophysiol 1996;76(1):571-81.

Clark CR, Geffen GM, Geffen LB. Role of monoamine pathways in attention and effort: effects of clonidine and methylphenidate in normal adult humans. Psychopharmacology 1986;90(1):35-9.

Coull JT, Middleton HC, Robbins TW, Sahakian BJ. Clonidine and diazepam have differential effects on tests of attention and learning. Psychopharmacology 1995;120(3):322-32.

Eisenach JC, Hood DD, Curry R. Intrathecal, but not intravenous, clonidine reduces experimental thermal or capsaicin-induced pain and hyperalgesia in normal volunteers. Anesth Analges 1998;87(3):591-6.

Eisenach JC, Hood DD, Curry R. Relative potency of epidural to intrathecal clonidine differs between acute thermal pain and capsaicin-induced allodynia. Pain 2000;84(1):57-64.

Frot M, Rambaud L, Guenot M, Mauguiere F. Intracortical recordings of early pain-related $\mathrm{CO} 2$-laser evoked potentials in the human second somatosensory (SII) area. Clin Neurophysiol 1999;110(1): 133-45.

Fuchs M, Drenckhahn R, Wischmann HA, Wagner M. An improved boundary element method for realistic volume-conductor modeling. IEEE Trans Biomed Eng 1998;45(8):980-97.

Hao JX, Yu W, Xu XJ, Wiesenfeld-Hallin Z. Effects of intrathecal vs. systemic clonidine in treating chronic allodynia-like response in spinally injured rats. Brain Res 1996;736(1-2):28-34.

Hari R, Hämäläinen M, Kaukoranta E, Reinikainen K, Teszner D. Neuromagnetic responses from the second somatosensory cortex in man. Acta Neurol Scand 1983a;68(4):207-12.

Hari R, Kaukoranta E, Reinikainen K, Huopaniemie T, Mauno J. Neuromagnetic localization of cortical activity evoked by painful dental stimulation in man. Neurosci Lett 1983b;42(1):77-82.

Hämäläinen H, Hiltunen J, Titievskaja I. fMRI activations of SI and SII cortices during tactile stimulation depend on attention. Neuroreport 2000;11(8):1673-6.

Hillebrand A, Barnes GR. A quantitative assessment of the sensitivity of whole-head MEG to activity in the adult human cortex. NeuroImage 2002;16:638-50.

Höchstetter K, Rupp A, Meinck HM, Weckesser D, Bornfleth H, Stippich C, et al. Magnetic source imaging of tactile input shows task-independent attention effects in SII. Neuroreport 2000;11(11): $2461-5$.

Howland EW, Wakai RT, Mjaanes BA, Balog JP, Cleeland CS. Whole head mapping of magnetic fields following painful electric finger shock. Cogn Brain Res 1995;2(3):165-72. 
Kakigi R, Koyoma S, Hoschiyama M, Kitamura Y, Shimolo M, Watanabe S. Pain-related magnetic fields following painfull $\mathrm{CO} 2$ laser stimulation in man. Neurosci Lett 1995;192(1):45-8.

Kakigi R, Koyoma S, Hoschiyama M, Kitamura Y, Shimojo M, Watanabe S, et al. Effects of taktile interference stimulation on somatosensory evoked magnetic fields. Neuroreport 1996;7(1): 405-8.

Kakigi R, Hoschiyama M, Shimojo M, Naka D, Yamasaki H, Watanabe S, et al. The somatosensory evoked magnetic fields. Prog Neurobiol 2000;61(5):495-523.

Kanui TI, Tjolsen A, Lund A, Mjellem-Joly N, Hole K. Antinociceptive effects of intrathecal administration of alpha-adrenoceptor antagonists and clonidine in the formalin test in the mouse. Neuropharmacology 1993;32(4):367-71.

Laudahn R, Kohlhoff H, Bromm B. Magnetoencephalography in the investigation of cortical pain processing. In: Bromm B, Desmedt JH, editors. Pain and the Brain, From Nociception to Cognition. NY: Raven Press; 1995. p. 267-81.

Lenz FA, Rios M, Chau D, Krauss GL, Zirh TA, Lesser RP. Painful stimuli evoke potentials recorded from the parasylvian cortex in humans. J Neurophysiol 1998;80(4):2077-88.

Lorenz J, Hauck M, Paur RC, Nakamura Y, Zimmermann R, Bromm $\mathrm{B}$, et al. Cortical correlates of false expectations during pain intensity judgments - a possible manifestation of placebo/nocebo cognitions. Brain Behav Immun 2005;19(4):281-2.

Melzack R, Casey KL. Localized temperature changes evoked in the brain by somatic stimulation. Exp Neurol 1967;17(3): 276-92.
Mima T, Nagamine T, Nakamura K, Shibasaki H. Attention modulates both primary and second somatosensory cortical activities in humans: a magnetoencephalographic study. J Neurophysiol 1998;80(4):2215-21.

Nakamura Y, Paur R, Zimmermann R, Bromm B. The attentional modulation of human pain processing in the secondary somatosensory cortex. A magneto-encephalographic study. Neurosci Lett 2002;328(1):29-32.

Quante M, Scharein E, Zimmermann R, Langer-Brauburger B, Bromm B. Dissociation of morphine analgesia and sedation evaluated by EEG measures in healthy volunteers. Arzneimittelforschung 2004;54(3):143-51.

Sarter M, Givens B, Bruno JP. The cognitive neuroscience of sustained attention: where top-down meets bottom-up. Brain Res Brain Res Rev 2001;35(2):146-60.

Scharein E, Bromm B. The intracutaneous pain model in the assessment of analgesic efficacy. Pain Reviews 1998;5:216-46.

Talairach J, Tournoux P. Co-Planar Stereotaxic Atlas of the Human Brain. Stuttgart, New York: Thieme; 1988.

Talbot JD, Marrett S, Evans AC, Meyer E, Bushnell MC, Duncan GH. Multiple representations of pain in human cerebral cortex. Science 1991;251(4999):1355-8.

Treede RD, Meyer RA, Raja SN, Campbell JN. Evidence for two different heat transduction mechanisms in nociceptive primary afferents innervating monkey skin. J Physiol 1995;483(Pt 3):747-58.

Treede RD, Apkarian AV, Bromm B, Greenspan JD, Lenz FA. Cortical representation of pain: functional characterization of nociceptive areas near the lateral sulcus. Pain 2000;87(2):113-9. 